\title{
Is Paradigm for the Role of Balloon-Assisted Enteroscopy Changing in Crohn's Disease?
}

\author{
Yoo Jin Lee \\ Division of Gastroenterology and Hepatology, Department of Internal Medicine, Keimyung University School of Medicine, Daegu, Korea
}

Corresponding Author

Yoo Jin Lee

ORCID https://orcid.org/0000-0003-1799-0146

E-mail doctorlyj@gmail.com
See "Enteroscopy in Crohn's Disease: Are There Any Changes in Role or Outcomes Over Time? A KASID Multicenter Study" by Seong Ran Jeon, et al. on page 375, Vol. 15, No. 3, 2021
Crohn's disease (CD) commonly involves the small bowel. The occurrence of small-bowel CD (SBCD) has been reported in over $60 \%$ of the patients with $\mathrm{CD} .{ }^{1}$ As SBCD is often associated with poor clinical outcomes, such as complicated disease course, stricturing behavior, and CDrelated surgery, early diagnosis and objective evaluation of small bowel lesions are essential. ${ }^{2}$ However, the evaluation of SBCD is more difficult than that of colonic lesions because the diseased segment of most small bowel lesions is difficult to reach during conventional endoscopy, and may appear normal on a cross-sectional image. ${ }^{2,3}$ Thus, the diagnosis of SBCD is challenging and often delayed.

Since the introduction of balloon-assisted enteroscopy (BAE) in the early 2000s, the evaluation of small bowel lesions has revolutionized dramatically. ${ }^{4}$ Unlike capsule endoscopy or other imaging modalities, BAE allows direct inspection of the small bowel mucosa, histopathological investigation by obtaining biopsies, and management of complications, such as stricture dilatation or hemostasis. In addition, diagnostic BAE has been shown to have a favorable diagnostic yield of up to $70 \%$ and low complication rate (approximately $1 \%){ }^{5}$ Despite being a useful tool, the widespread use of BAE was hampered soon after its implementation, as it is an invasive procedure that requires advanced skills and laborious procedure time.

Based on the recommendations of the Selecting Therapeutic Targets in Inflammatory Bowel Disease (STRIDE) working group, a treat-to-target strategy is now a key concept in the treatment of inflammatory bowel disease. ${ }^{6}$ A treat-to-target strategy, which means continuous adjustment of treatment based on proactive monitoring and targeting mucosal healing rather than symptoms, seems to be helpful in limiting disease progression and improving clinical outcomes. ${ }^{7}$ In line with this concept, the importance of adequate evaluation of small bowel lesions has been emphasized. ${ }^{2}$ As BAE has been accepted to be the most sensitive modality for visualization of small bowel lesions in $\mathrm{CD}$, it may enable not only a more objective diagnosis but also close monitoring of SB lesions in patients with $\mathrm{CD}$. Thus, recently published Japanese guidelines also stated that BAE may be useful for close observation and monitoring of SB lesions in $\mathrm{CD}{ }^{8}$

In the current issue, Jeon et al. ${ }^{9}$ reported data comparing BAE-related factors and clinical outcomes of BAE use in SBCD over time. From A multicenter database of patients with $\mathrm{CD}$ with small bowel lesions who underwent BAE, 131 procedures in 116 patients were included. After dividing into the first and second periods based on the introduction of the BAE guidelines in 2008, enteroscopic findings, diagnostic yield, treatment, and clinical impact of BAE were compared. The most common indication for BAE was the diagnosis of small bowel involvement during each period ( $50 \%$ vs $31.3 \%, \mathrm{p}=0.034$ ), and nearly twothirds of all patients adjusted their therapeutic plan after BAE. These results suggest that BAE plays an important role in the diagnosis and monitoring of SBCD in clinical settings.

Meanwhile, despite the drastic advances in the medical treatment of $\mathrm{CD}$, therapy for SBCD stricture remains a representative unmet need. Up to $75 \%$ of patients with $\mathrm{CD}$ ultimately undergo surgery at least once during their lifetime, and the main cause is strictures. ${ }^{10}$ Stricture dilatation 
with BAE has been shown to have a high technical success rate $(94.9 \%)$ and a low rate of major complications $(5.3 \%){ }^{10}$ Consistent with the result of previous reports, the study by Jeon et al. ${ }^{9}$ also showed a high technical success rate of over $80 \% .{ }^{9}$ One of the most notable findings of this study is that the use of BAE related to small bowel strictures was significantly increased in the second period compared to that in the first period $(2.9 \%$ vs $21.3 \%, \mathrm{p}=0.002)$. This finding indicates that $\mathrm{BAE}$ is expanding its scope to diagnosis as well as treatment of SBCD. Nevertheless, we should pay attention to the fact that the reported long-term clinical outcomes of stricture dilatation have been shown to be more favorable in patients who underwent surgery than in those with stricture dilatation through BAE. ${ }^{3}$ Thus, although stricture dilatation through BAE can replace or delay surgery, more experience and evidence are needed to select the best candidates for BAE intervention in real clinical practice.

This study was limited by the lack of long-term followup of clinical outcomes; however, the findings are meaningful as they have shown the changes in procedure indications and clinical outcomes of BAE in patients with $\mathrm{CD}$ with small bowel involvement over time. As the treatment concept of CD shifts from symptom-based therapy to treat-to-target strategy, BAE is expected to play a more important role in the diagnosis as well as close monitoring and therapeutic intervention of SBCD in the near future.

\section{CONFLICTS OF INTEREST}

No potential conflict of interest relevant to this article was reported.

\section{ORCID}

Yoo Jin Lee https://orcid.org/0000-0003-1799-0146

\section{REFERENCES}

1. Park SH, Kim YJ, Rhee KH, et al. A 30-year trend analysis in the epidemiology of inflammatory bowel disease in the Songpa-Kangdong district of Seoul, Korea in 1986-2015. J Crohns Colitis 2019;13:1410-1417.

2. Watanabe K. Clinical management for small bowel of Crohn's disease in the treat-to-target era: now is the time to optimize treatment based on the dominant lesion. Intest Res 2020;18:347-354.

3. Yoo JH, Holubar S, Rieder F. Fibrostenotic strictures in Crohn's disease. Intest Res 2020;18:379-401.

4. Yamamoto H, Sekine Y, Sato Y, et al. Total enteroscopy with a nonsurgical steerable double-balloon method. Gastrointest Endosc 2001;53:216-220.

5. Heine GD, Hadithi M, Groenen MJ, Kuipers EJ, Jacobs MA, Mulder CJ. Double-balloon enteroscopy: indications, diagnostic yield, and complications in a series of 275 patients with suspected small-bowel disease. Endoscopy 2006;38:4248.

6. Peyrin-Biroulet L, Sandborn W, Sands BE, et al. Selecting Therapeutic Targets in Inflammatory Bowel Disease (STRIDE): determining therapeutic goals for treat-to-target. Am J Gastroenterol 2015;110:1324-1338.

7. Colombel JF, D’haens G, Lee WJ, Petersson J, Panaccione R. Outcomes and strategies to support a treat-to-target approach in inflammatory bowel disease: a systematic review. J Crohns Colitis 2020;14:254-266.

8. Nakase H, Uchino M, Shinzaki S, et al. Evidence-based clinical practice guidelines for inflammatory bowel disease 2020 . J Gastroenterol. Epub 2021 Apr 22. https://doi.org/10.1007/ s00535-021-01784-1.

9. Jeon SR, Kim JO, Byeon JS, et al. Enteroscopy in Crohn's disease: are there any changes in role or outcomes over time? A KASID Multicenter Study. Gut Liver 2021;15:375-382.

10. Bettenworth D, Bokemeyer A, Kou L, et al. Systematic review with meta-analysis: efficacy of balloon-assisted enteroscopy for dilation of small bowel Crohn's disease strictures. Aliment Pharmacol Ther 2020;52:1104-1116. 